\title{
43. PALYGORSKITE, SEPIOLITE, AND OTHER CLAY MINERALS IN LEG 41 OCEANIC SEDIMENTS: MINERALOGY, FACIES, AND GENESIS
}

\author{
P.P. Timofeev, V.V. Eremeev, and M.A. Rateev, \\ Geological Institute of the USSR Academy of Sciences, Moscow, USSR
}

\section{INTRODUCTION}

Leg 41 holes, drilled near the northwest margin of Africa, penetrated Mesozoic and Cenozoic sediments. The $<1 \mu \mathrm{m}$ fraction of these sediments contains montmorillonite, kaolinite, hydromica, chlorite, mixedlayer minerals of the mica-montmorillonite and chlorite-montmorillonite types, palygorskite, and sepiolite.

\section{STRATIGRAPHIC AND LITHOLOGIC OCCURRENCE OF MAGNESIAN SILICATES}

Occurrence of magnesian silicates of the palygorskite and sepiolite type proved widespread and is shown in Figure 1. These minerals occur in the Albian of Site 368 and the Cenomanian of Site 370. Palygorskite frequently occurs with sepiolites; this is unusual for platform deposits. Palygorskite also occurs in Eocene sediments: lower Eocene of Hole 366, lower and middle Eocene of Hole 370, middle Eocene of Hole 369, and upper Eocene of Hole 368; in Hole 367, palygorskite occurs in Upper Cretaceous and lower Eocene sediments.

Magnesian silicates are associated with the following lithologies (Figure 2):

1) Clay and claystone with gentle wavelike laminations (Hole 368, Upper Cretaceous to lower Eocene).

2) Claystone with interlayers of sandy silty material (Hole 370, lower to middle Eocene; Hole 368, upper Eocene).

3) Nannofossil marl alternating with claystone and siltstone (Hole 370, Cenomanian to upper Paleocene).

4) Nannofossil marl with burrows (Hole 369, Albian).

5) Limestone with interbeds of clay (Hole 369, middle Eocene; Hole 366, lower Eocene).

Tables 1 through 5 and Figure 2 show that in Holes 366 and 369 , magnesian silicates are associated with organogenic clay and carbonate sediments, whereas those of Holes 368 and 370 are associated with terrigenous and, at Site 368 , with partly chemogenic sediments.

\section{MICROSCOPIC FEATURES OF PALYGORSKITIC AND PALYGORSKITIC-SEPIOLITIC ROCKS}

Clays and claystones of nearly pure palygorskiticsepiolitic composition occur as dim, dark gray, doublerefracted masses. These masses occur with fine grains, plates of brown biotite, remains of plants, spores and pollen, globules of pyrite, and pyritized cherty fossils.

The clay mass is frequently filled with rhombohedrons of diagenetic dolomite. The texture of clays is flocculent, cirrose, cellular, or lumpy. Because of similar optical features, palygorskite and sepiolite are indistinguishable from each other in thin section. Finegrained terrigenous material is negligible in clays of this type; we recognized no volcanogenic material. Palygorskitic monomineral clays usually contain a greater admixture of quartz and are better recrystallized.

Claystones with interbeds of silty material consist of elongated plates of hydromica and scaly, slightly double-refracted masses of palygorskite-kaolinite, which cement angular grains of silt-size quartz. Authigenic rhombohedrons of dolomite permeate the entire rock.

Limestone containing palygorskite is composed of a pelitomorphic mass of calcite that includes organic remains: coccoliths, foraminifer tests filled with clay and cherty matter, diatoms, and radiolarians. Palygorskite may occur throughout the rock (Sample 34-3, $83-85 \mathrm{~cm}$ ), or less frequently as independent aggregates (Sample 35-3, 104-106 cm).

\section{MINERALOGY OF SEPIOLITES AND PALYGORSKITES}

We could not determine the chemical composition of sepiolites in Leg 41 cores, because they occur with palygorskite or other minerals. Palygorskite of Leg 41 deposits shows intense X-ray peaks which differentiate it readily from sepiolite (Mumpton and Roy, 1958):

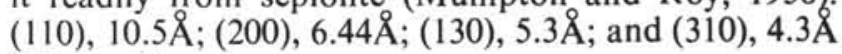
(Figure 3).

The palygorskite DTA scans are characterized by absence of an exothermic peak at $860^{\circ} \mathrm{C}$ (as in sepiolite), lower temperature of the mid-endothermal effect at $490-510^{\circ} \mathrm{C}$, and by more intense endothermic peaks at $255^{\circ}-270^{\circ} \mathrm{C}$.

The oceanic palygorskites are similar in composition to those of the Russian platform (Table 6). They contain up to $10 \% \mathrm{Al}_{2} \mathrm{O}_{3}$ and over $9 \% \mathrm{MgO}$, which is representative of the Russian platform. The $\mathrm{TiO}_{2}$ content in palygorskites is about the same as in other clays. In sepiolite, however, $\mathrm{TiO}_{2}$ is usually absent; this is regarded as additional proof of authigeneity. The carbonate content in palygorskitic clays does not exceed $1 \%$, and organic carbon is only $0.42 \%$; this is representative of palygorskitic clays of the arid zone. The iron sulfide content is $0.44 \%$.

\section{Possibility of Isomorphic Substitutions}

There are differing viewpoints concerning the possibility of isomorphic transformation of palygorskite into sepiolite, and vice-versa. Sepiolite and palygorskite may be intermediate members of the iso- 
P. P. TIMOFEEV, V. V. EREMEEV, M. A. RATEEV
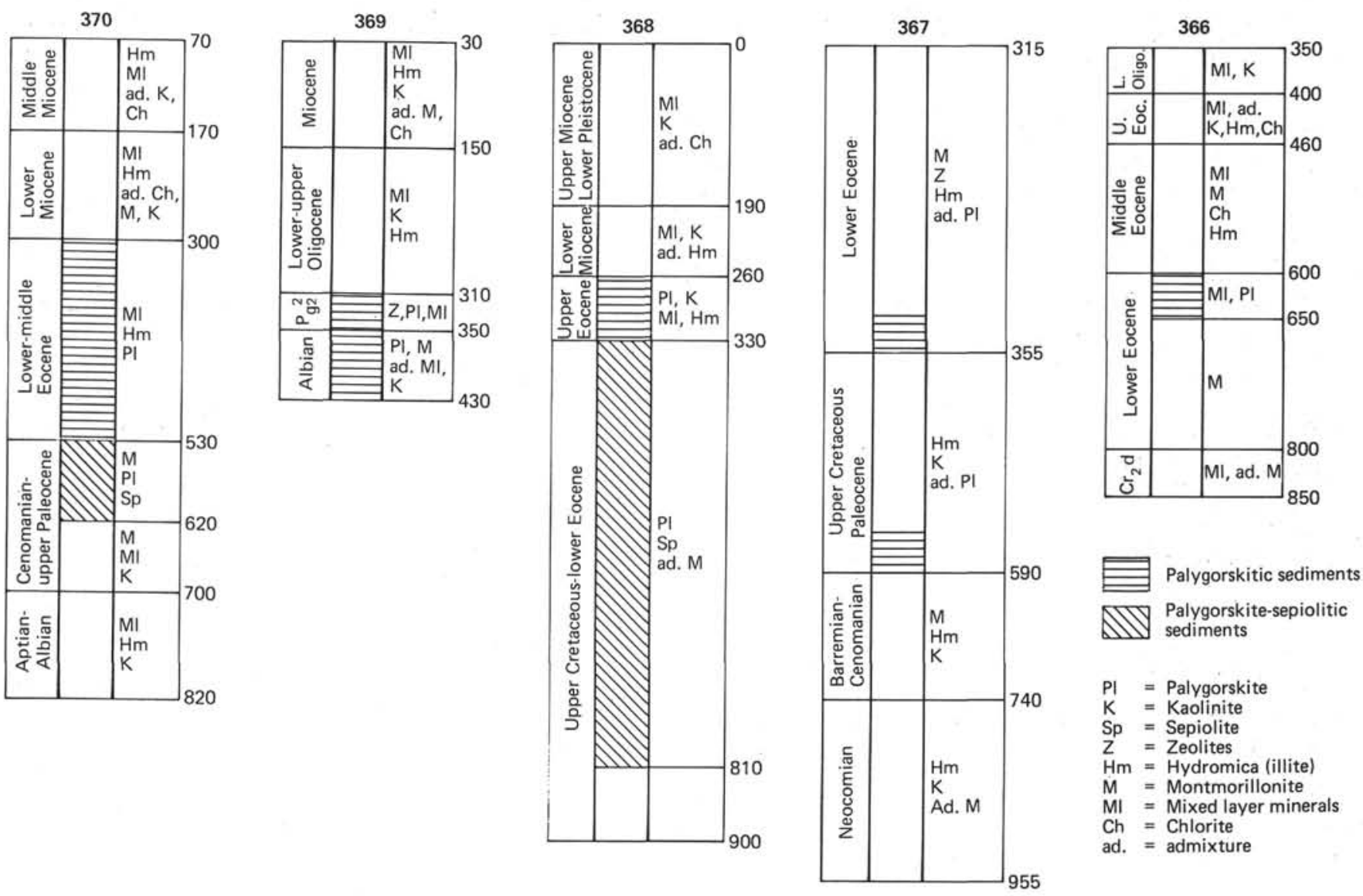

Figure 1. Occurrence of palygorskite and sepiolite in Leg 41 sediments.
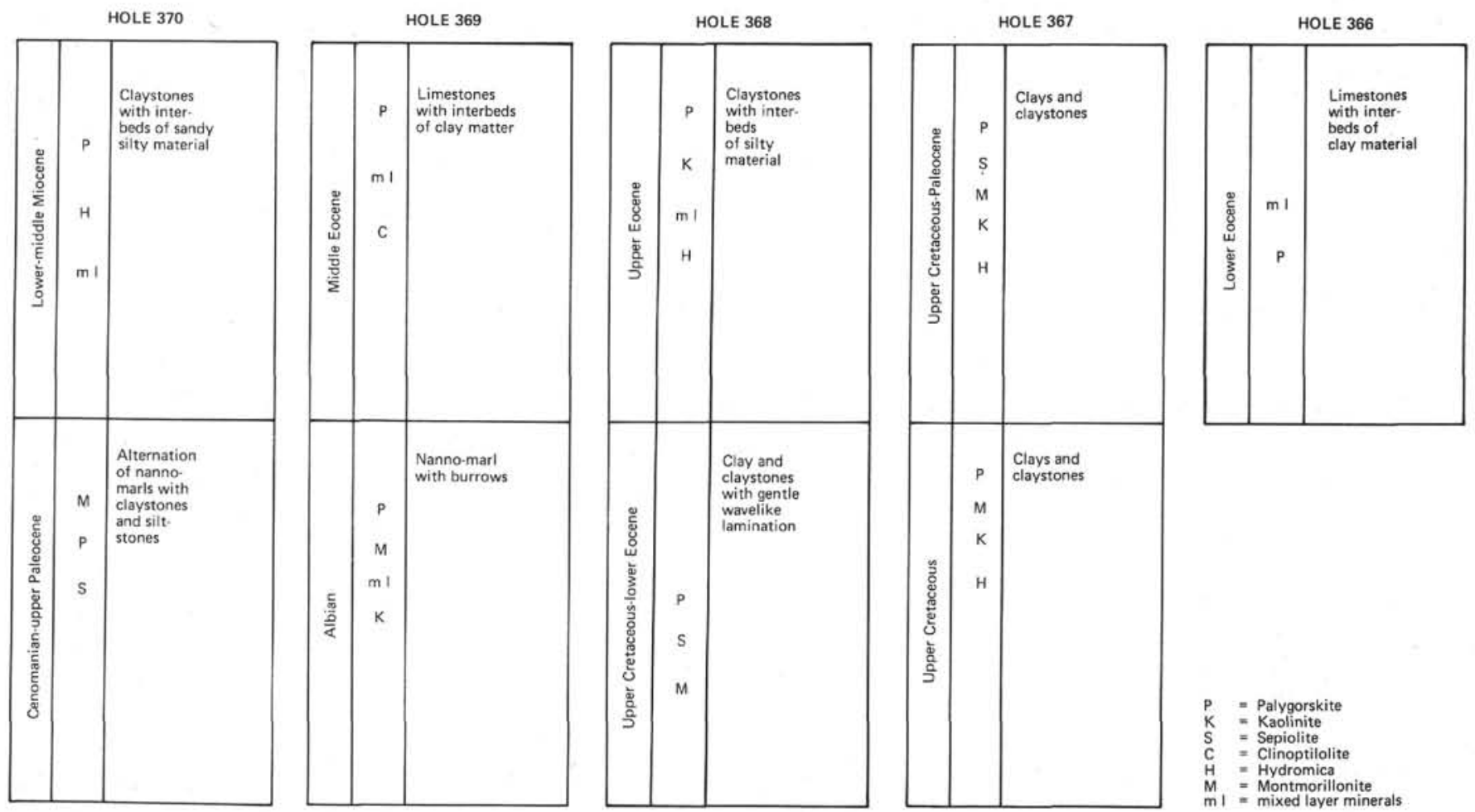

Figure 2. Lithologic associations of magnesian silicates in Leg 41 sediments. 
TABLE

Clay Minerals in Hole 366

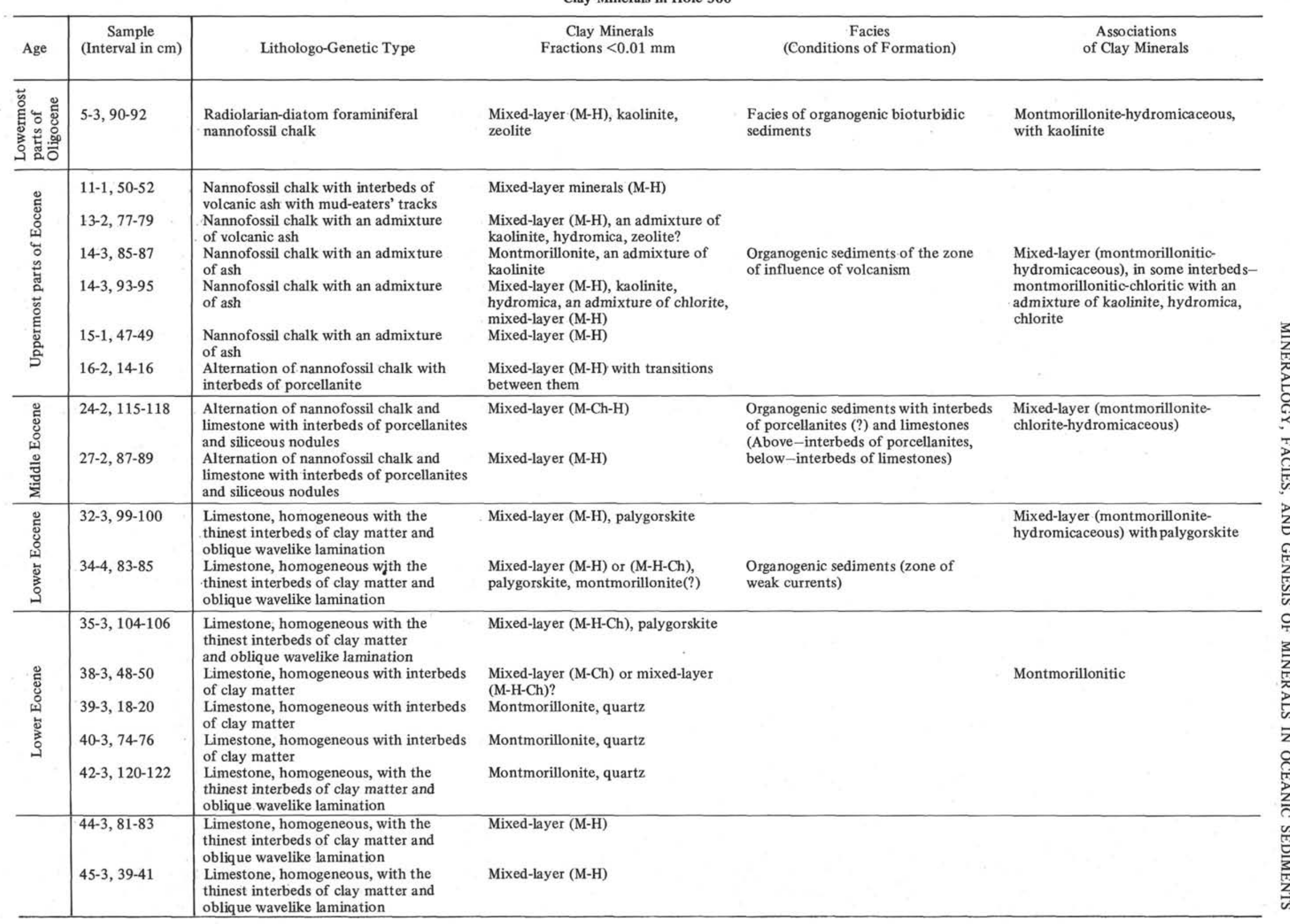




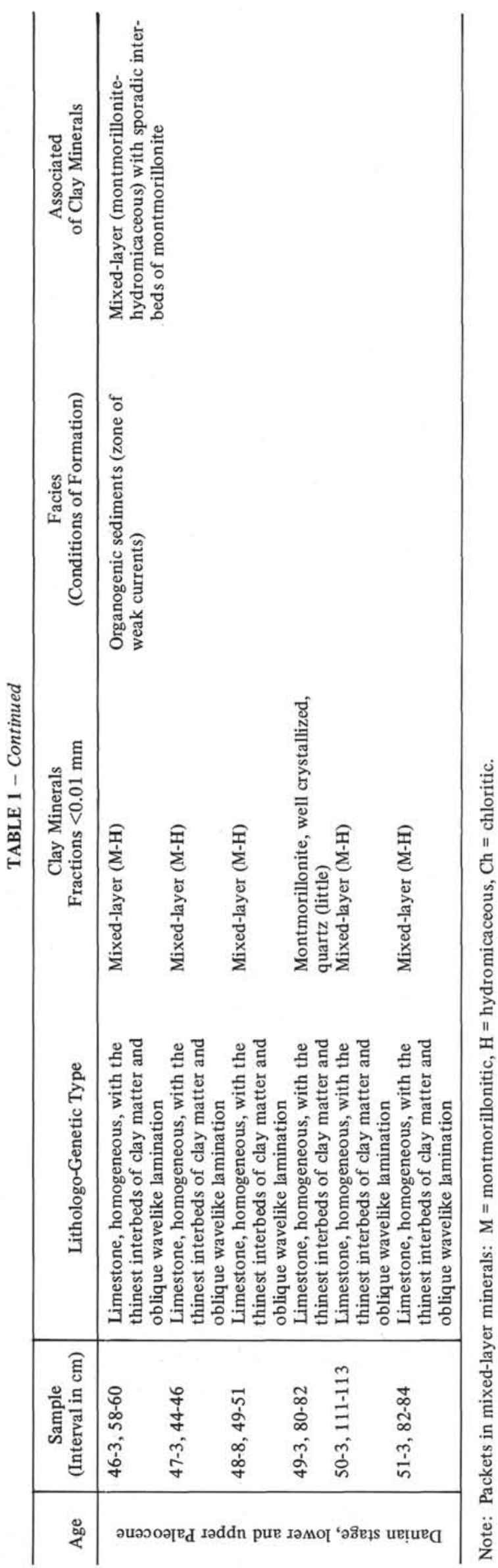

morphic series talc-pyrophyllite, with progressive substitution of $\mathrm{Al}$ for $\mathrm{Mg}$ positions in octahedral layers of the montmorillonite type. Martin-Vivaldi and CanoRuiz (1956) have supported this idea. But in comparing chemical compositions of sepiolite and palygorskite in Leg 41 sedimentary rocks, we recognized no reliable features of isomorphism. All Russian-platform carboniferous sepiolites that we studied, from both the arid zone (middle and upper Carboniferous) and the humid zone (lower Carboniferous), were completely pure. Chemical analyses of sepiolites, after exclusion of carbonates, yielded typical compositions (Table 2). Rare deviations from the standard composition resulted from admixtures of other minerals. Chambers (1959) found a thick bed of sepiolite in the sedimentary deposits of Vallecas (Spain) to be homogeneous. Thus, we are unable to find any transitional members on the basis of the $\mathrm{Al}$ and $\mathrm{Mg}$ contents of sedimentary bedded sepiolites and palygorskites.

Studies by Nagi and Bradley (1955) and by Preisinger (1959) showed significant differences in the structures of sepiolite and palygorskite. Palygorskites may be regarded as intermediate minerals-with compositional extremes $\mathrm{Mg}_{3} / \mathrm{OH}_{2} \mathrm{Si}_{4} \mathrm{O}_{10}$ (magnesian-montmorillonitic) and $\mathrm{Al}_{2} / \mathrm{OH}_{2} \mathrm{Si}_{4} \mathrm{O}_{10}$ (aluminum-montmorillonitic) - between talc and pyrophyllite. Substitution of $\mathrm{Al}$ for $\mathrm{Mg}$ can be of three types (after S.G. Dromeshko, personal communication), within the following molecular ratios:

$$
\begin{aligned}
& \mathrm{Mg}: \mathrm{Al}=2: 1 \\
& \mathrm{Mg}: \mathrm{Al}=1: 1 \\
& \mathrm{Mg}: \mathrm{Al}=1: 2
\end{aligned}
$$

In palygorskite from the Russian platform we were able to establish only two types: $\mathrm{Mg}: \mathrm{Al}=1: 1$ and 2:1 (Table 7), without any gradual transitions. Leg 41 palygorskite is of the second type (Table 7).

\section{ENVIRONMENT OF SEPIOLITE AND PALYGORSKITE FORMATION}

Magnesian silicates occur most abundantly in clay rocks of Holes 368 and 370, particularly in sections where material was supplied from Africa. The terrigenous material derives from the Senegal River basin; the coarse fraction suggests deposition by turbidity currents, but does not indicate local volcanic sources.

The occurrence of palygorskitic-sepiolitic rocks in Hole 370 is related to an underwater canyon or delta. The Lower Cretaceous to middle Eocene section of Hole 370 has the character of the underwater debris cone from the lower continental slope. Sedimentary textures, contacts of layers, and grain-size distribution in coarse clastic layers confirm that from the Lower Cretaceous to the upper Tertiary, coarse clastic material was deposited by turbidity currents.

Magnesian silicate clays occur less abundantly in organogenic carbonate-clay rocks of Holes 366 and 369. In Hole 369 , palygorskite is related to pelagic sediments deposited near the continental slope of the undrained coast during the Eocene arid climate. Coarser terrigenous material is absent because this region received no sediment from northwest Africa 
TABLE 2

Clay Minerals in Hole 367

\begin{tabular}{|c|c|c|c|c|c|}
\hline Age & $\begin{array}{c}\text { Sample } \\
\text { (Interval in } \mathrm{cm} \text { ) }\end{array}$ & Lithologo-Genetic Type & $\begin{array}{c}\text { Clay Mineral } \\
\text { Fraction }<0.01 \mathrm{~mm}\end{array}$ & $\begin{array}{c}\text { Facies Zone } \\
\text { (Condition of Formation) }\end{array}$ & $\begin{array}{c}\text { Association } \\
\text { of Clay Minerals }\end{array}$ \\
\hline Pliocene & $4-3,54-56$ & $\begin{array}{l}\text { Foraminifer-radiolarian- } \\
\text { containing nannofossil marl } \\
\text { with interbeds of silty } \\
\text { material }\end{array}$ & $\begin{array}{l}\text { Montmorillonite, kaolinite } \\
\text { with hydromica }\end{array}$ & $\begin{array}{l}\text { Organogenic-clay sediments } \\
\text { (zone of quiet sedimentation) }\end{array}$ & Montmorillonite \\
\hline Early Eocene & $14-3,18-20$ & Clay zeolite containing & $\begin{array}{l}\text { Na-montmorillonite } \\
\text { clinoptillolite }\end{array}$ & $\begin{array}{l}\text { Clay sediments (zone of } \\
\text { quiet sedimentation) }\end{array}$ & $\begin{array}{l}\text { Montmorillonite- } \\
\text { zeolite with } \\
\text { sepiolite }\end{array}$ \\
\hline $\begin{array}{l}\text { Paleocene- } \\
\text { Late } \\
\text { Cretaceous }\end{array}$ & $15-3,70-72$ & Silty clay & $\begin{array}{l}\text { K-montmorillonite }(\sim 30 \%) \\
\text { kaolinite }(\sim 30 \%) \\
\text { hydromica }(\sim 30 \%) \text { with } \\
\text { palygorskite }\end{array}$ & $\begin{array}{l}\text { Silty-clay sediment (zone of } \\
\text { a steep slope) }\end{array}$ & $\begin{array}{l}\text { Montmorillonite- } \\
\text { kaolinite- } \\
\text { hydromica with } \\
\text { palygorskite and } \\
\text { sepiolite }\end{array}$ \\
\hline $\begin{array}{l}\text { Late } \\
\text { Cretaceous }\end{array}$ & $16-3,118-120$ & Silty clay & $\begin{array}{l}\text { Na-montmorillonite } \\
\text { kaolinite } \\
\text { hydromica }\end{array}$ & $\begin{array}{l}\text { Silty-clay sediment (zone of } \\
\text { a steep slope) }\end{array}$ & $\begin{array}{l}\text { Montmorillonite- } \\
\text { kaolinite- } \\
\text { hydromica with } \\
\text { palygorskite }\end{array}$ \\
\hline $\begin{array}{l}\text { Late } \\
\text { Cretaceous }\end{array}$ & $17-3,92-94$ & $\begin{array}{l}\text { Claystone with } \\
\text { horizontal lamination }\end{array}$ & $\begin{array}{l}\mathrm{Na}, \mathrm{Mg}, \mathrm{Ca}-\text { montmorillonite } \\
\text { hydromica with } \\
\text { palygorskite and } \\
\text { kaolinite }\end{array}$ & $\begin{array}{l}\text { Silty-clay sediment (zone of } \\
\text { a steep slope) }\end{array}$ & \\
\hline $\begin{array}{l}\text { Early Albian } \\
\text { late Aptian }\end{array}$ & $23-2,64-66$ & $\begin{array}{l}\text { Claystone with interbeds } \\
\text { of fine-grained siltstones }\end{array}$ & $\begin{array}{l}\text { Montmorillonite with } \\
\text { hydromica and kaolinite }\end{array}$ & $\begin{array}{l}\text { Silty-clay sediment (zone of } \\
\text { a steep slope) }\end{array}$ & \\
\hline $\begin{array}{l}\text { Early Aptian- } \\
\text { Barremian }\end{array}$ & $\begin{array}{l}24-3,79-81 \\
24, \mathrm{CC}\end{array}$ & $\begin{array}{l}\text { Claystone with interbeds } \\
\text { of fine-grained siltstones }\end{array}$ & $\begin{array}{l}\begin{array}{l}\text { Na-montmorillonite } \\
\text { hydromica, kaolinite }\end{array} \\
\text { Mg, Ca-montmorillonite } \\
\text { hydromica }(20 \%)\end{array}$ & $\begin{array}{l}\text { Silty-clay sediment (zone of } \\
\text { a steep slope) } \\
\text { Silty-clay sediment (zone of } \\
\text { a steep slope) }\end{array}$ & $\begin{array}{l}\text { Montmorillonite } \\
\text { with hydromica } \\
\text { and kaolinite }\end{array}$ \\
\hline $\begin{array}{l}\text { Early } \\
\text { Cretaceous }\end{array}$ & $28-2,73-75$ & $\begin{array}{l}\text { Limestones with interbeds } \\
\text { of clays }\end{array}$ & $\begin{array}{l}\text { Hydromica (illite) } \\
\text { kaolinite }(20 \%-30 \%)\end{array}$ & $\begin{array}{l}\text { Organogenic sediments with } \\
\text { interbeds of clay matter } \\
\text { (zone of quiet sedimentation) }\end{array}$ & $\begin{array}{l}\text { Hydromica- } \\
\text { kaolinite with } \\
\text { montmorillonite }\end{array}$ \\
\hline
\end{tabular}

during Cretaceous and Tertiary time (Seibold and Hinz, 1974). Clastic material was trapped and deposited in the Gulf of Tarfaya, and only clay was transported to the continental slope.

In the region of Hole 366, on the Sierra Leone Rise, Cenozoic sediments are largely open-marine pelagic facies, resulting from open-ocean microfauna in the absence of any terrigenous material except eolian. On the Sierra Leone Rise, the middle Miocene sediments were subject to conditions similar to those at present. Palygorskite probably could have been supplied to the pelagic sediments (Holes 366 and 369) only through eolian transport. This accords with the diverse accompanying clay minerals-montmorillonite, mixedlayer minerals, kaolinite, etc. (Table 4)-in clayey interbeds of palygorskitic limestones.

\section{Transfer and Redeposition of Palygorskite and Sepiolite Particles}

As to genesis of clays, Millot (1964) suggests that particles of magnesium silicate cannot survive transportation. Other sediments indicate, however, that there has been stable redeposition by fluvial processes or deep-sea currents. Goldberg and Griffin (1970) discuss eolian transfer of clay particles. Thus, clay particles can be transported in many ways: by wind, river runoff, redeposition as result of shore- and bottom-abrasion, and oceanic currents. Transport of palygorskite and sepiolite in turbulent currents is therefore probable.

\section{Supply of Palygorskite and Sepiolite From Northwest Africa}

Elouard (1959) studied Eocene sediments north of the Senegal River basin, in the region of the mouth of the Senegal, and in the territory of Mauritania-i.e., near Hole 368 . Here, the basement rocks are transgressively overlain only by middle Eocene deposits. These epicontinental deposits reflect chemogenic sedimentation with formation of limestone, dolomite, chert, and palygorskitic clay containing montmorillonite. Elouard (1959) showed that $65 \mathrm{~km}$ from the boundary of the basin, the clay fraction is composed entirely of palygorskite. Toward the boundary of the basin, palygorskite is replaced by montmorillonite. Near the shoreline, both are replaced by detrital kaolinite brought in with sandy material.

Palygorskitic-sepiolitic rocks occur in Dahomey, Ivory Coast, Nigeria, Niger, Gabon, the Sudan, and others. This testifies to the regional importance of the epochs of palygorskite formation on the African continent. Coincidence of the epochs of palygorskite formation in oceanic and epicontinental sediments should not be overlooked. The oldest occurrence is at the top of Cretaceous (Maestrichtian), the maximum is in the Eocene, and the third occurrence is in the Miocene.

\section{Genesis of Palygorskite and Sepiolite in Leg 41 Oceanic Sediments}

Analysis of genetic and facies types of palygorskitebearing deposits shows that genesis of these magnesian 
TABLE 3

Clay Minerals in Hole 368

\begin{tabular}{|c|c|c|c|c|c|}
\hline Age & $\begin{array}{c}\text { Sample } \\
\text { (Interval in } \mathrm{cm} \text { ) }\end{array}$ & Lithologo-Genetic Type & Clay Mineral Fractions $<0.01 \mathrm{~mm}$ & $\begin{array}{c}\text { Facies Zone } \\
\text { (Conditions of Formation) }\end{array}$ & $\begin{array}{l}\text { Associations of } \\
\text { Clay Minerals }\end{array}$ \\
\hline 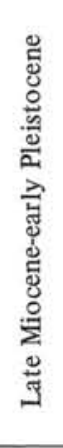 & $\begin{array}{l}5-4,73-75 \\
6-5,75-77 \\
7-5,90-92 \\
8-4,72-74 \\
9-4,83-84\end{array}$ & $\begin{array}{l}\text { Nannofossil oozes and nannomarls } \\
\text { Nannofossil oozes and nannomarls } \\
\text { Nannofossil oozes and nannomarls } \\
\text { Nannofossil oozes and nannomarls }\end{array}$ & $\begin{array}{l}\text { Mixed-layer (M-H), kaolinite } \\
\text { Mixed-layer (M-H), kaolinite } \\
\text { Mixed-layer (M-H-Ch), kaolinite, a small } \\
\text { admixture of chlorite } \\
\text { Mixed-layer (M-H), kaolinite, an } \\
\text { admixture of hydromica and mont- } \\
\text { morillonite } \\
\text { Mixed-layer (M-H-CH) }\end{array}$ & $\begin{array}{l}\text { Organogenic sediments (zone of quiet } \\
\text { sedimentation) (alternation of nanno- } \\
\text { fossil oozes with nannofossil marls) }\end{array}$ & $\begin{array}{l}\text { Mixed-layer (montmorillonite- } \\
\text { hydromicaceous with a varying ratio } \\
\text { of packets) with kaolinite, less } \\
\text { frequently chlorite }\end{array}$ \\
\hline 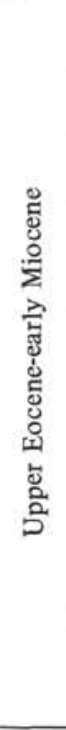 & $\begin{array}{l}17-4,54-57 \\
18-4,85-87 \\
22-5,88-90\end{array}$ & $\begin{array}{l}\text { Claystone, horizontally laminated } \\
\text { with interbeds of sandy-silty } \\
\text { material } \\
\text { Claystone, horizontally laminated } \\
\text { with interbeds of sandy-silty } \\
\text { material } \\
\text { Claystone, horizontally laminated } \\
\text { with interbeds of sandy-silty } \\
\text { material } \\
\text { Claystone, horizontally laminated } \\
\text { with interbeds of sandy-silty } \\
\text { material } \\
\text { Claystone, horizontally laminated } \\
\text { with interbeds of sandy-silty } \\
\text { material } \\
\text { Claystone, horizontally laminated } \\
\text { with interbeds of sandy-silty } \\
\text { material } \\
\text { Clay, brown, horizontally- } \\
\text { laminated, enriched with } \\
\text { zeolite }\end{array}$ & $\begin{array}{l}\text { Mixed-layer (M-H), kaolinite, } \\
\text { admixture of hydromica } \\
\text { Mixed-layer (Ch-M-H), kaolinite } \\
\text { Palygorskite, kaolinite, mixed-layer } \\
\text { (M-H) } \\
\text { Montmorillonite, kaolinite, hydromica }\end{array}$ & $\begin{array}{l}\text { Organogenic-clay sediments of the } \\
\text { quiet sedimentation zone (in the upper } \\
\text { part mostly organogenic-clayey, and } \\
\text { in the lower-more clayey sediments } \\
\text { with an admixture of silty material) }\end{array}$ & $\begin{array}{l}\text { Kaolinite-mixed-layer (montmorillonite- } \\
\text { hydromicaceous), sometimes with } \\
\text { palygorskite }\end{array}$ \\
\hline 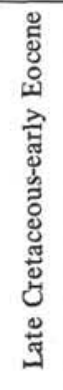 & $\begin{array}{l}25-2,65-67 \\
26-2,80-82 \\
29-1,68-70 \\
30-2,58-60 \\
31-2,78-80 \\
34-2,61-62 \\
36-2,38-40 \\
36-3,37-39\end{array}$ & $\begin{array}{l}\text { Claystone, horizontally laminated } \\
\text { Claystone, horizontally laminated } \\
\text { Claystone, horizontally laminated } \\
\text { Claystone, horizontally laminated } \\
\text { Claystone, horizontally laminated } \\
\text { Claystone, horizontally laminated } \\
\text { Claystone with gentle wavelike } \\
\text { small discontinuous lamination } \\
\text { Claystone with gentle wavelike } \\
\text { small discontinuous lamination }\end{array}$ & $\begin{array}{l}\text { Palygorskite, a small admixture of } \\
\text { montmorillonite } \\
\text { Palygorskite, a small admixture of } \\
\text { montmorillonite } \\
\text { Montmorillonite, palygorskite } \\
\text { Palygorskite, mixed-layer (M-H) } \\
\text { Palygorskite } \\
\text { Palygorskite } \\
\text { Palygorskite, sepiolite } \\
\text { Palygorskite, sepiolite }\end{array}$ & & Palygorskite-montmorillonitic \\
\hline
\end{tabular}




\begin{tabular}{|c|c|c|c|c|c|}
\hline 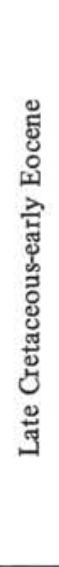 & $\begin{array}{l}37-4,80-82 \\
39-5,69-70 \\
40-3,69-70 \\
41-2,70-72 \\
42-2,62-63\end{array}$ & $\begin{array}{l}\text { Claystone with gentle wavelike } \\
\text { small discontinuous lamination } \\
\text { Claystone with gentle wavelike } \\
\text { small discontinuous lamination } \\
\text { Claystone with gentle wavelike } \\
\text { small discontinuous lamination } \\
\text { Claystone with gentle wavelike } \\
\text { small discontinuous lamination } \\
\text { Claystone with gentle wavelike } \\
\text { small discontinuous lamination } \\
\text { Claystone with gentle wavelike } \\
\text { small discontinuous lamination } \\
\text { Claystone with gentle wavelike } \\
\text { small discontinuous lamination } \\
\text { Claystone with gentle wavelike } \\
\text { small discontinuous lamination } \\
\text { Claystone with gentle wavelike } \\
\text { small discontinuous lamination }\end{array}$ & $\begin{array}{l}\text { Sepiolite, palygorskite } \\
\text { Sepiolite, palygorskite } \\
\text { Palygorskite, montmorillonite or } \\
\text { mixed-layer (M-H) } \\
\text { Sepiolite, palygorskite, montmorillonite } \\
\text { or montmorillonite-hydromica } \\
\text { Sepiolite, palygorskite, montmorillonite, } \\
\text { quartz } \\
\text { Sepiolite, palygorskite, montmorillonite }\end{array}$ & $\begin{array}{l}\text { Deep-water clay sediments with dolomite } \\
\text { (zone of quiet sedimentation); in the } \\
\text { lower part (layers } 39-47 \text { ) zones of weak } \\
\text { currents with a small admixture of silty } \\
\text { material and areas of discontinuous } \\
\text { wavelike lamination }\end{array}$ & $\begin{array}{l}\text { Palygorskite-sepiolite-montmorillonite- } \\
\text { mixed-layer }\end{array}$ \\
\hline & $\begin{array}{l}58-5,68-70 \\
59-3,88-90\end{array}$ & $\begin{array}{l}\text { Alternation of thinly elutriated } \\
\text { claystone with a sandy-silty } \\
\text { variety, gentle wavelike small } \\
\text { discontinuous lamination } \\
\text { Alternation of thinly elutriated } \\
\text { claystone with a sandy-silty } \\
\text { variety, gentle wavelike small } \\
\text { discontinuous lamination } \\
\text { Alternation of thinly elutriated } \\
\text { claystone with a sandy-silty } \\
\text { variety, gentle wavelike small } \\
\text { discontinuous lamination } \\
\text { Alternation of thinly elutriated } \\
\text { claystone with a sandy-silty } \\
\text { variety, gentle wavelike small } \\
\text { discontinuous lamination } \\
\text { Alternation of thinly elutriated } \\
\text { claystone with a sandy-silty } \\
\text { variety, gentle wavelike small } \\
\text { discontinuous lamination } \\
\text { Alternation of thinly elutriated } \\
\text { claystone with a sandy-silty } \\
\text { variety, gentle wavelike small } \\
\text { discontinuous lamination } \\
\text { Silty clay, cryptohorizontally } \\
\text { laminated } \\
\text { Claystone, horizontally laminated } \\
\text { with interbeds of coaly claystone } \\
\text { Alternation of claystone interbeds } \\
\text { and sandy-silty varieties }\end{array}$ & $\begin{array}{l}\text { Montmorillonite, hydromica, mixed- } \\
\text { layer (M-H), kaolinite (traces) } \\
\text { Hydromica, mixed-layer (M-H), chlorite } \\
\text { kaolinite } \\
\text { Hydromica, mixed-layer (M-H), } \\
\text { chlorite, quartz, feldspars }\end{array}$ & $\begin{array}{l}\text { Siltstone-clay sediments (zone of weak } \\
\text { currents) }\end{array}$ & $\begin{array}{l}\text { Hydromicaceous-kaolinitic, sometimes } \\
\text { with an admixture of mixed-layer } \\
\text { (montmorillonite-hydromicaceous) } \\
\text { minerals }\end{array}$ \\
\hline
\end{tabular}

Deep-water clay sediments with dolomite dmixture of silt material and areas of discontinuous velike lamination

Note: Packets in mixed-layer minerals; $\mathrm{M}=$ montmorillonitic, $\mathrm{H}=$ hydromicaceous, $\mathrm{Ch}=$ chloritic. 
TABLE 4

Clay Minerals in Hole 369

\begin{tabular}{|c|c|c|c|c|c|}
\hline Age & $\begin{array}{c}\text { Sample } \\
\text { (Interval in } \mathrm{cm} \text { ) }\end{array}$ & Lithologo-Genetic Type & $\begin{array}{l}\text { Clay Mineral } \\
\text { Fractions }<0.01 \mathrm{~mm}\end{array}$ & $\begin{array}{c}\text { Facies } \\
\text { (Conditions of Formation) }\end{array}$ & $\begin{array}{l}\text { Associations of } \\
\text { Clay Minerals }\end{array}$ \\
\hline \multirow{10}{*}{ 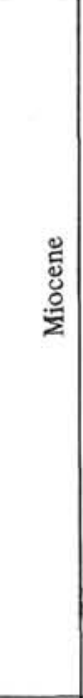 } & $1-3,84-86$ & $\begin{array}{l}\text { Nannofòssil oozes, homogeneous, } \\
\text { cryptohorizontally laminated }\end{array}$ & $\begin{array}{l}\text { Hydromica, mixed-layer (H-M), } \\
\text { admixture of chlorite and kaolinite }\end{array}$ & \multirow{10}{*}{$\begin{array}{l}\text { Organogenic-clay sediments } \\
\text { (zone of influence of volcanism) }\end{array}$} & \multirow{10}{*}{$\begin{array}{l}\text { Mixed-layer-hydromicaceous } \\
\text { kaolinitic (sometimes with an } \\
\text { admixture of hydromica, } \\
\text { chlorite, or montmorillonite) }\end{array}$} \\
\hline & $2-3,82-84$ & $\begin{array}{l}\text { Nannofossil oozes, homogeneous, } \\
\text { cryptohorizontally laminated }\end{array}$ & Mixed-layer (H-M), kaolinite & & \\
\hline & $2-4,80-81$ & $\begin{array}{l}\text { Nannofossil oozes, homogeneous } \\
\text { cryptohorizontally laminated }\end{array}$ & $\begin{array}{l}\text { Mixed-layer (H-M), hydromica, } \\
\text { kaolinite }\end{array}$ & & \\
\hline & $3-2,73-75$ & $\begin{array}{l}\text { Nannofossil oozes, homogeneous, } \\
\text { cryptohorizontally laminated }\end{array}$ & $\begin{array}{l}\text { Mixed-layer (H-M), hydromica, } \\
\text { kaolinite }\end{array}$ & & \\
\hline & $3-3,80-82$ & $\begin{array}{l}\text { Nannofossil oozes, homogeneous, } \\
\text { cryptohorizontally laminated }\end{array}$ & $\begin{array}{l}\text { Montmorillonite, kaolinite, } \\
\text { chlorite, mixed-layer }(\mathrm{Ch}-\mathrm{H})\end{array}$ & & \\
\hline & $4-2,92-94$ & $\begin{array}{l}\text { Nannofossil oozes, homogeneous, } \\
\text { cryptohorizontally laminated }\end{array}$ & $\begin{array}{l}\text { Mixed-layer }(\mathrm{M}-\mathrm{H} \text { and } \mathrm{Ch}-\mathrm{H}) \text {, } \\
\text { kaolinite, quartz }\end{array}$ & & \\
\hline & $4-4,80-82$ & $\begin{array}{l}\text { Nannofossil oozes, homogeneous, } \\
\text { cryptohorizontally laminated }\end{array}$ & $\begin{array}{l}\text { Hydromica, mixed-layer }(\mathrm{M}-\mathrm{H}) \text {, } \\
\text { kaolinite, quartz }\end{array}$ & & \\
\hline & $4-5,84-86$ & $\begin{array}{l}\text { Nannofossil marl with interbeds of clay } \\
\text { enriched with ash }\end{array}$ & $\begin{array}{l}\text { Mixed-layer (M-H and } \mathrm{Ch}-\mathrm{H}) \text {, } \\
\text { hydromica,kaolinite }\end{array}$ & & \\
\hline & $6-2,73-75$ & $\begin{array}{l}\text { Nannofossil marl with interbeds of clay } \\
\text { enriched with ash }\end{array}$ & $\begin{array}{l}\text { Mixed-layer (M-H and } \mathrm{Ch}-\mathrm{H}) \text {, } \\
\text { hydromica, kaolinite, a small } \\
\text { admixture of chlorite }\end{array}$ & & \\
\hline & $7-5,84-86$ & $\begin{array}{l}\text { Nannofossil marl with interbeds of clay } \\
\text { enriched with ash }\end{array}$ & $\begin{array}{l}\text { Mixed-layer (M-H and } \mathrm{Ch}-\mathrm{H}) \text {, hydro- } \\
\text { mica, kaolinite, an admixture of } \\
\text { chlorite }\end{array}$ & & \\
\hline \multirow{4}{*}{ 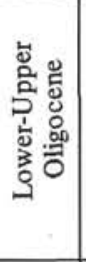 } & & $\begin{array}{l}\text { Nannofossil marl with abundance of } \\
\text { mud-eaters' tracks }\end{array}$ & $\begin{array}{l}\text { Kaolinite, mixed-layer (M-H), } \\
\text { hydromica }\end{array}$ & \multirow{4}{*}{$\begin{array}{l}\text { Organogenic-clay sediments } \\
\text { (reworking zone } \\
\text { by bottom organisms) }\end{array}$} & \multirow[t]{4}{*}{$\begin{array}{l}\text { Mixed-layer-kaolinitic- } \\
\text { hydromicaceous }\end{array}$} \\
\hline & $26-4,70-72$ & $\begin{array}{l}\text { Nannofossil marl with abundance of } \\
\text { mud-eaters' tracks }\end{array}$ & $\begin{array}{l}\text { Mixed-layer (M-H), montmorillonite, } \\
\text { kaolinite, hydromica }\end{array}$ & & \\
\hline & $27-5,91-93$ & $\begin{array}{l}\text { Nannofossil marl with abundance of } \\
\text { mud-eaters' tracks }\end{array}$ & $\begin{array}{l}\text { Mixed-layer (M-H), kaolinite, } \\
\text { hydromica }\end{array}$ & & \\
\hline & $31-4,80-81$ & $\begin{array}{l}\text { Nannofossil marl with abundance of } \\
\text { mud-eaters' tracks }\end{array}$ & $\begin{array}{l}\text { Mixed-layer (M-H and H-M), } \\
\text { kaolinite }\end{array}$ & & \\
\hline \multirow{2}{*}{ 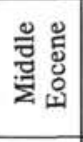 } & $34-2,78-81$ & $\begin{array}{l}\text { Limestone, homogeneous with } \\
\text { interbeds of clay matter }\end{array}$ & Mixed-layer (M-H), zeolite & \multirow[t]{2}{*}{$\begin{array}{l}\text { Organogenic sediments } \\
\text { (zone of quiet sedimentation) }\end{array}$} & \multirow[t]{2}{*}{$\begin{array}{l}\text { Zeolite-palygorskitic- } \\
\text { mixed-layer }\end{array}$} \\
\hline & $35-2,78-81$ & $\begin{array}{l}\text { Limestone, homogeneous with } \\
\text { interbeds of clay matter }\end{array}$ & Palygorskite, zeolite & & \\
\hline \multirow{4}{*}{ 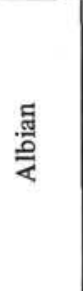 } & $40-4,83-85$ & $\begin{array}{l}\text { Nannofossil marl with rare mud-eaters' } \\
\text { tracks, cryptohorizontally laminated }\end{array}$ & $\begin{array}{l}\text { Montmorillonite, palygorskite, a } \\
\text { a small admixture of kaolinite, } \\
\text { quartz }\end{array}$ & \multirow{4}{*}{$\begin{array}{l}\text { Organogenic-clay sediments } \\
\text { (zone of quiet sedimentation) }\end{array}$} & \multirow{4}{*}{$\begin{array}{l}\text { Palygorskite-montmorillonitic } \\
\text { (sometimes an admixture of } \\
\text { mixed-layer minerals and } \\
\text { kaolinite) }\end{array}$} \\
\hline & $41-4,80-82$ & $\begin{array}{l}\text { Nannofossil marl with rare mud-eaters' } \\
\text { tracks, cryptohorizontally laminated }\end{array}$ & $\begin{array}{l}\text { Montmorillonite, an admixture of } \\
\text { palygorskite }\end{array}$ & & \\
\hline & $42-1,79-81$ & $\begin{array}{l}\text { Nannofossil marl with rare mud-eaters' } \\
\text { tracks, cryptohorizontally laminated }\end{array}$ & Palygorskite, mixed-layer $(\mathrm{H}-\mathrm{Ch})$ & & \\
\hline & $49-4,69-70$ & $\begin{array}{l}\text { Nannofossil marl with rare mud-eaters' } \\
\text { tracks, cryptohorizontally laminated }\end{array}$ & $\begin{array}{l}\text { Palygorskite, mixed-layer (M-H), } \\
\text { a small admixture of kaolinite }\end{array}$ & & \\
\hline
\end{tabular}

Note: Packets in mixed-layer minerals: $\mathrm{M}=$ montmorillonitic, $\mathrm{H}=$ hydromicaceous, $\mathrm{Ch}=$ chloritic. The first component of the associations is given in the largest amounts. 
TABLE 5

Clay Minerals in Hole 370

\begin{tabular}{|c|c|c|c|c|c|}
\hline Age & $\begin{array}{c}\text { Sample } \\
\text { (Interval in } \mathrm{cm} \text { ) }\end{array}$ & Lithologo-Genetic Type & $\begin{array}{c}\text { Clay Mineral } \\
\text { Fractions }<0.01 \mathrm{~mm}\end{array}$ & $\begin{array}{c}\text { Facies } \\
\text { (Conditions of Formation) }\end{array}$ & $\begin{array}{c}\text { Association } \\
\text { of Clay Minerals }\end{array}$ \\
\hline 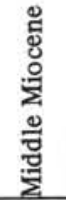 & $\begin{array}{l}4-2,68-70 \\
4-3,110-120\end{array}$ & $\begin{array}{l}\text { Claystone, silty with interbeds of } \\
\text { coarse silty and fine aranaceous } \\
\text { material } \\
\text { Claystone, silty with interbeds of } \\
\text { coarse silty and fine aranaceous } \\
\text { material }\end{array}$ & $\begin{array}{l}\text { Hydromica, mixed-layer (H-M), an } \\
\text { admixture of chlorite } \\
\text { Hydromica, mixed-layer (Ch-H) } \\
\text { and (H-M), an admixture of kaolinite }\end{array}$ & $\begin{array}{l}\text { Silty-clayey sediments } \\
\text { (zone of quiet sedimentation) }\end{array}$ & $\begin{array}{l}\text { Hydromicaceous-mixed-layer, } \\
\text { with an admixture of kaolinite, }\end{array}$ \\
\hline 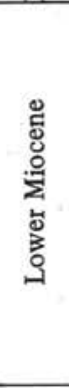 & $\begin{array}{l}5-3,35-36 \\
5-4,97-99 \\
8-2,64-66\end{array}$ & $\begin{array}{l}\text { Claystone, silty with interbeds of } \\
\text { coarse silty and fine aranaceous } \\
\text { material } \\
\text { Claystone, silty with interbeds of } \\
\text { coarse silty and fine aranaceous } \\
\text { material } \\
\text { Claystone, silty with interbeds of } \\
\text { coarse silty and fine aranaceous } \\
\text { material } \\
\text { Claystone, silty, with small oblique } \\
\text { not unidirectional and gentle } \\
\text { wavelike lamination }\end{array}$ & $\begin{array}{l}\text { Hydromica, mixed-layer (H-Ch), an } \\
\text { admixture of chlorite and kaolinite } \\
\text { Mixed-layer (M-H) and (Ch-H), } \\
\text { hydromica, an admixture of chlorite, } \\
\text { kaolinite? } \\
\text { Mixed-layer (M-H and Ch-H), hydro- } \\
\text { mica, chlorite } \\
\text { Montmorillonite, mixed-layer (Ch-H), } \\
\text { an admixture of chlorite }\end{array}$ & $\begin{array}{l}\text { Silty-clayey sediments } \\
\text { (zone of weak currents) }\end{array}$ & $\begin{array}{l}\text { Mixed-layer-hydromicaceous, } \\
\text { with an admixture of chlorite, } \\
\text { montmorillonite, kaolinite? }\end{array}$ \\
\hline 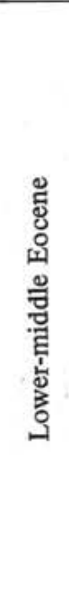 & $\begin{array}{l}10-2,88-90 \\
12-1,113-115 \\
14-4,99-100 \\
15-2,98-100 \\
16-2,17-18 \\
17-2,141-143\end{array}$ & $\begin{array}{l}\text { Claystone, silty, with small oblique } \\
\text { not unidirectional and gentle } \\
\text { wavelike lamination } \\
\text { Claystone, silty, with interbed s of } \\
\text { coarse-silty and fine-arenaceous } \\
\text { material with oblique lamination } \\
\text { Claystone, silty, with interbeds of } \\
\text { coarse-silty and fine-aranaceous } \\
\text { material with oblique lamination } \\
\text { Claystone, silty, with interbeds of } \\
\text { coarse-silty and fine-aranaceous } \\
\text { material with oblique lamination } \\
\text { Claystone, silty, with interbeds of } \\
\text { coarse-silty and fine-aranaceous } \\
\text { material with oblique lamination } \\
\text { Alternation of interbeds of coarse- } \\
\text { grained siltstone with nannofossil } \\
\text { containing marls and claystone with } \\
\text { oblique intertruncated lamination }\end{array}$ & $\begin{array}{l}\text { Mixed-layer (M-H and Ch-H), hydro- } \\
\text { mica, chlorite, palygorskite } \\
\text { Mixed-layer (H-M), hydromica, } \\
\text { chlorite, palygorskite } \\
\text { Mixed-layer (M-H), hydromica, } \\
\text { chlorite } \\
\text { Mixed-layer (M-H) and (H-Ch) } \\
\text { Mixed-layer (M-H and H-M) a small } \\
\text { admixture of chlorite } \\
\text { Mixed-layer (M-H), and admixture of } \\
\text { palygorskite }\end{array}$ & $\begin{array}{l}\text { Silty-clayey sediments } \\
\text { (zone of weak sea currents) }\end{array}$ & $\begin{array}{l}\text { Mixed-layer-hydromicaceous- } \\
\text { palygorskitic }\end{array}$ \\
\hline
\end{tabular}




\begin{tabular}{|c|c|c|c|c|c|}
\hline Age & $\begin{array}{c}\text { Sample } \\
\text { (Interval in } \mathrm{cm} \text { ) }\end{array}$ & Lithologo-Genetic Type & $\begin{array}{c}\text { Clay Mineral } \\
\text { Fractions }<0.01 \mathrm{~mm}\end{array}$ & $\begin{array}{c}\text { Facies } \\
\text { (Conditions of Formation) }\end{array}$ & $\begin{array}{c}\text { Association } \\
\text { of Clay Minerals }\end{array}$ \\
\hline 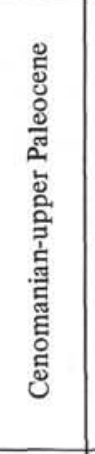 & $\begin{array}{l}19-1,91-93 \\
20-2,68-71 \\
23-4,100-102\end{array}$ & $\begin{array}{l}\text { Alternation of interbeds of coarse- } \\
\text { grained siltstone with nannofossil } \\
\text { containing marls and claystone with } \\
\text { oblique intertruncated lamination } \\
\text { Alternation of interbeds of coarse- } \\
\text { grained siltstone with nannofossil } \\
\text { containing marls and claystone with } \\
\text { oblique intertruncated lamination } \\
\text { Alternation of interbeds of silty } \\
\text { marl and claystone with oblique, } \\
\text { intertruncated lamination } \\
\text { Alternation of interbeds of silty } \\
\text { marl and claystone with oblique, } \\
\text { intertruncated lamination }\end{array}$ & $\begin{array}{l}\text { Montmorillonite, palygorskite, sepiolite, } \\
\text { chlorite }\end{array}$ & $\begin{array}{l}\text { Silty-clayey sediments } \\
\text { (zone of quiet sedimentation) }\end{array}$ & $\begin{array}{l}\text { Montmorillonite-mixed-layer } \\
\text { with kaolinite }\end{array}$ \\
\hline 䃾 & $\begin{array}{l}24-3,86-88 \\
24-5,18-19 \\
26-3,90-92 \\
32-4,68-70\end{array}$ & $\begin{array}{l}\text { Claystone, nannofossil containing, with } \\
\text { interbeds of silty-arenaceous material } \\
\text { with oblique intertruncated lamination } \\
\text { Claystone, nannofossil containing, with } \\
\text { interbeds of silty-arenaceous material } \\
\text { with oblique intertruncated lamination } \\
\text { Claystone, nannofossil containing, with } \\
\text { interbeds of silty-arenaceous material } \\
\text { with oblique intertruncated lamination } \\
\text { Claystone, silty, with interbeds of } \\
\text { coarse-silty and fine-arenaceous } \\
\text { material with oblique intertruncated } \\
\text { lamination }\end{array}$ & $\begin{array}{l}\text { Mixed-layer }(M-H), \text { kaolinite } \\
\text { Mixed-layer (M-H), hydromica, } \\
\text { kaolinite } \\
\text { Mixed-layer (M-H), hydromica, } \\
\text { kaolinite }\end{array}$ & $\begin{array}{l}\text { Silty-clayey sediments } \\
\text { (zone of weak sea currents) }\end{array}$ & $\begin{array}{l}\text { Mixed-layer-hydromicaceous- } \\
\text { kaolinitic }\end{array}$ \\
\hline 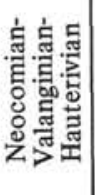 & $\begin{array}{l}34-4,68-71 \\
44-2,40-42\end{array}$ & $\begin{array}{l}\text { Alternation of nannofossil containing } \\
\text { clay stone and siltstone with oblique } \\
\text { intertruncated lamination } \\
\text { Nannofossil containing marl alternating } \\
\text { with interbeds of silty material with } \\
\text { oblique intertruncated lamination }\end{array}$ & $\begin{array}{l}\text { Hydromica, montmorillonite, kaolinite, } \\
\text { chlorite } \\
\text { Hydromica, mixed-layer }(\mathrm{Ch}-\mathrm{H}) \\
\text { kaolinite }\end{array}$ & $\begin{array}{l}\text { Silty-clayey sediments } \\
\text { (zone of currents in the } \\
\text { deltaic area) }\end{array}$ & $\begin{array}{l}\text { Hydromica-montmorillonite- } \\
\text { kaolinitic }\end{array}$ \\
\hline
\end{tabular}

Note: Packets in mixed-layer minerals: $\mathrm{M}=$ montmorillonitic, $\mathrm{H}=$ hydromicaceous, $\mathrm{Ch}=$ chloritic. The first components in the associations have the largest amounts. 
silicate minerals can differ even within the lithologic section of one hole. In continental facies of Africa, palygorskites are related chiefly to carbonate sediments. Likewise, in Leg 41 sediments the most significant amounts of palygorskite occur with slightly carbonaceous clays and claystones.

Comparatively pure monomineralic palygorskitic or sepiolitic clays, nearly devoid of terrigenous material and associated with some dolomite, are likely to form through a chemogenic or chemogenic-diagenetic process. Formation of these rocks in Holes 368 and 370 occurred at depths below the critical level of carbonate accumulation, very near the African continent. Chemogenic formation of palygorskite and sepiolite was possible here not under arid conditions, as usually occurs, but in an environment of normal marine sedimentation, owing to an intense inflow of dissolved $\mathrm{Mg}$ cations, possibly as $\mathrm{Mg}(\mathrm{OH})_{2}$, and of silica from the area of tropical weathering and laterite formation. ${ }^{1}$ Such weathering and laterization is known to have occurred on the African continent (Millot, 1964). Heating of the oceanic waters may also have been important near the continent.

Palygorskitic clays with admixtures of terrigenous particles and accompanying clay minerals could not have resulted from mineral selection during eolian or fluvial transport. Authigenic, chemogenic-diagenetic formation of palygorskitic clays therefore seems most probable.

The second type of palygorskitic clays and claystones, with interbeds of sandy-silty material (Holes 368 and 370), was formed by transfer of palygorskitic, lacustrine, and marine epicontinental sediments, probably as turbidity currents from the Senegal River delta (Hole 368) or from a submarine canyon (Hole 370 ) into the deep oceanic environment. The clay fraction usually contains palygorskite, montmorillonite, kaolinite, hydromica, mixed-layer minerals, occasional chlorite, etc. This is true in Lower Cretaceous sediments of Hole 370 and in upper Eocene to lower Miocene sediments of Holes 368 and 370. Millot (1964) recognized accompanying minerals in sediments of similar age in the continental facies of adjacent Africa. These palygorskitic-sepiolitic rocks of the arid belt are widely developed in northwest Africa; their thickness sometimes reaches 500 meters (Millot, 1964), and they would have been susceptible to wind erosion and transport to the ocean.

The third and fourth facies types of palygorskitebearing rocks are represented by organogenic limestones with interbeds of clay matter and nannofossil marls. These are typical pelagic sediments of an open sea or of an oceanic basin which bordered the undrained coast of Africa during the arid climate of Early Cretaceous and Eocene times. To explain their presence, the magnesian silicates of the regions of Holes 366 and 369 would seem to require epicontinental seas in the Late Cretaceous and the Eocene, with extremely shallow, strongly heated water, and chemogenic sedimentation of palygorskite-sepiolite, followed by

'Other evidence from Leg 41 suggests that $\mathrm{Mg}$ could also be supplied by brines emanating from evaporitic sediments deeper in the section. subsidence to a great depth. Or they could have been carried into these basins from the arid zone of Africa by wind.

The palygorskites in the clay fraction of pelagic sediments from Holes 366 and 369 are terrigenous, as confirmed by the presence of such minerals as montmorillonite, mixed-layer minerals, kaolinite, and quartz. Also, Pow-foong Fan and Rex (1972) say that in lower Pliocene to lower Miocene limey nannofossil oozes of Hole 136, palygorskite occurs with mica, montmorillonite, kaolinite, quartz, hematite, and chlorite in the $<2 \mu \mathrm{m}$ fraction. They give a similar association of minerals accompanying palygorskite, with small variations, for Mesozoic and Cenozoic sediments of Hole 135 and Holes 137 to 144 . Von Rad and Rösch (1972) also point out that in many samples of pelagic and hemipelagic clays from Leg 14, palygorskite is associated with montmorillonite, kaolinite, and quartz. Even in the Recent epoch, less favorable for palygorskite formation than the Cenozoic and Late Cretaceous, continent-to-ocean transfer of palygorskite particles occurs, as does the formation of palygorskite-bearing oceanic sediments.

TABLE 6

Chemical Composition of Magnesian Silicates of Oceanic Sediments of Carboniferous Deposits of the Mid-Carboniferous Russian Platform

\begin{tabular}{|c|c|c|c|c|c|}
\hline \multirow{4}{*}{$\begin{array}{l}\text { Components } \\
\mathrm{SiO}_{2}\end{array}$} & \multicolumn{3}{|c|}{ Palygorskite } & \multirow{3}{*}{\multicolumn{2}{|c|}{$\begin{array}{l}\text { Sepiolite } \\
\text { Platform }\end{array}$}} \\
\hline & Oceanic & & & & \\
\hline & $\begin{array}{c}\text { Hole } 368 \\
\text { Sample } 29-1,61-63 \\
\text { early Eocene }\end{array}$ & \multicolumn{2}{|c|}{ Platform } & & \\
\hline & 53.44 & 58.22 & 61.73 & 52.61 & 53.52 \\
\hline $\mathrm{TiO}_{2}$ & 0.48 & 0.45 & 0.43 & - & - \\
\hline $\mathrm{Al}_{2} \mathrm{O}_{3}$ & 9.60 & 10.71 & 11.01 & 1.58 & 1.64 \\
\hline $\mathrm{Fe}_{2} \mathrm{O}_{3}$ & 3.97 & 3.02 & 3.08 & 0.71 & 0.34 \\
\hline $\mathrm{FeO}$ & 0.44 & 0.33 & 0.33 & 0.36 & 0.44 \\
\hline $\mathrm{MgO}$ & 9.55 & 8.79 & 7.08 & 19.95 & 19.46 \\
\hline $\mathrm{CaO}$ & 2.36 & 1.03 & 0.50 & 4.82 & 8.47 \\
\hline $\mathrm{K}_{2} \mathrm{O}$ & 1.26 & 1.53 & 2.40 & 0.27 & 0.43 \\
\hline $\mathrm{Na}_{2} \mathrm{O}$ & 0.57 & 0.40 & 0.03 & 1.02 & 2.50 \\
\hline $\mathrm{H}_{2} \mathrm{O}_{-}^{-}$ & 8.36 & 8.56 & 7.30 & 6.72 & 4.31 \\
\hline $\mathrm{H}_{2}^{2} \mathrm{O}+$ & 9.97 & 6.96 & 6.11 & 11.96 & 8.89 \\
\hline Total & 100.00 & 100.00 & 100.00 & 100.00 & 100.00 \\
\hline
\end{tabular}

TABLE 7

Molecular Mg:Al Ratio in Oceanic and Russian Platform Palygorskites

\begin{tabular}{llc}
\hline Locality & \multicolumn{1}{c}{ Geological Age } & $\begin{array}{c}\text { Molecular } \\
\text { Ratio } \\
\text { Mg:Al }\end{array}$ \\
\hline $\begin{array}{l}\text { Hole } 368 \\
\text { Sample 29-1, 61-67 cm }\end{array}$ & Early Eocene & $1: 1$ \\
Russian Platform & $\begin{array}{l}\text { Middle Carboniferous } \\
\text { Kashirsky horizon } \mathrm{C}_{2}^{\mathrm{k}} \\
\text { Steshevsky horizon } \mathrm{C}_{2}^{\text {st }}\end{array}$ & $1: 1$ \\
& $\mathrm{C}_{2}^{\mathrm{k}}$ & $1: 1$ \\
$\mathrm{C}_{2}^{\mathrm{k}}$ & $2: 1$ \\
$\mathrm{C}_{2}^{\mathrm{k}}$ & $2: 1$ \\
$\mathrm{C}_{2}^{\mathrm{k}}$ & $2: 1$ \\
\hline
\end{tabular}


P. P. TIMOFEEV, V. V. EREMEEV, M. A. RATEEV
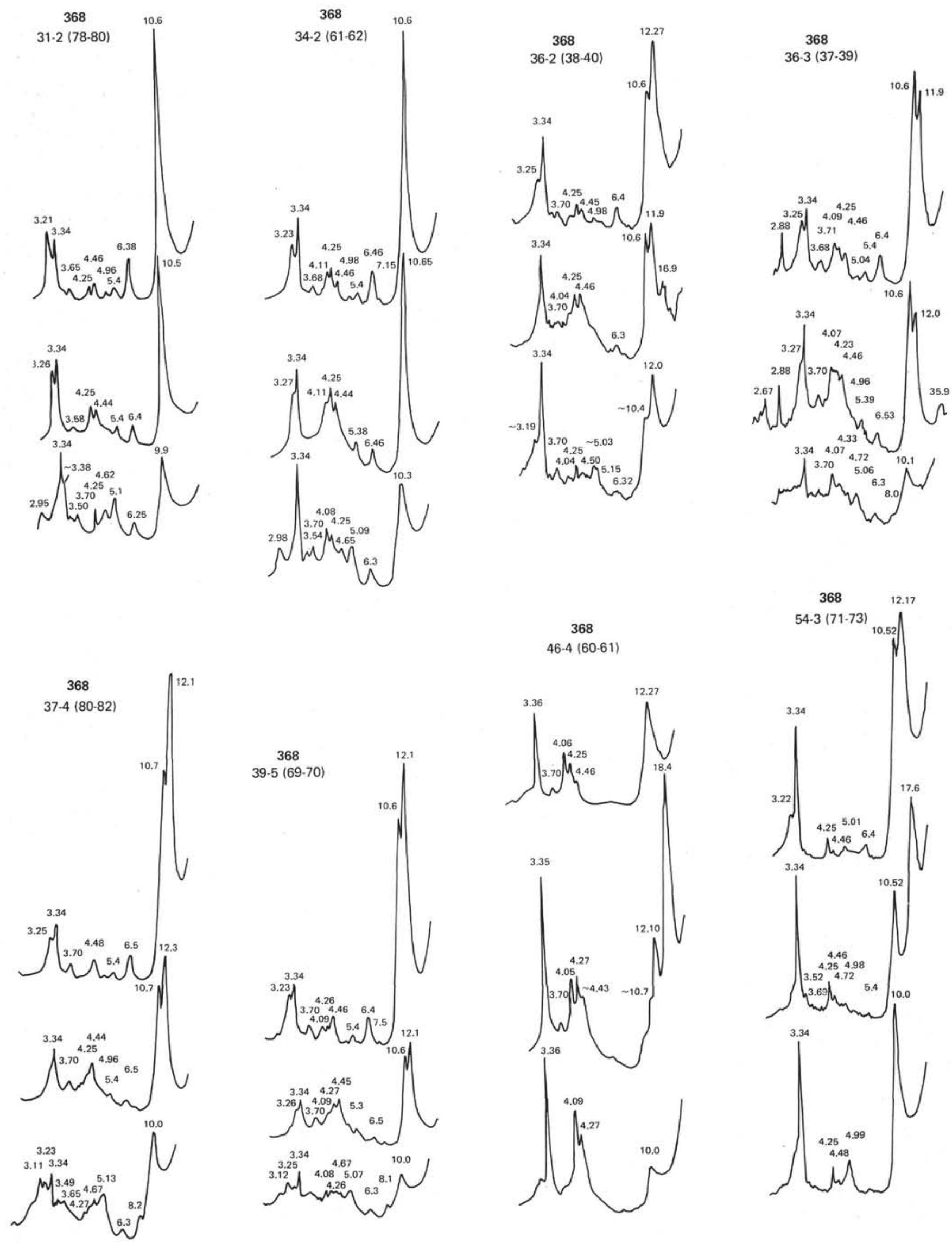

Figure 3. X-ray diffraction scans of Leg 41 magnesian silicates. 

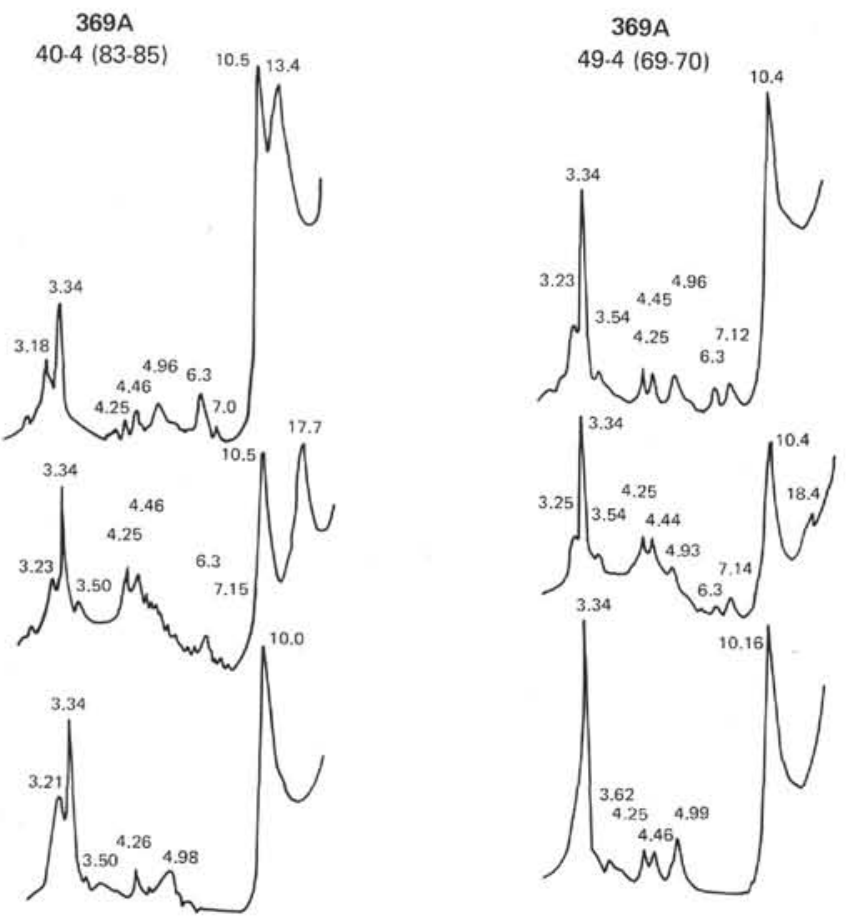

Figure 3. (Continued).

\section{Evidence Against Volcanic Origin of Palygorskite and Sepiolites in Leg 41 Sediments}

The following evidence argues against the assumed effect of hydrothermal flow of magnesian solutions: the occurrence of palygorskites and sepiolites in different facies; their occurrence in carbonate rocks peculiar to deep-sea red clays; monomineral palygorskite clays with no traces of hydrothermal metasomatic replacement, decoloration, or disturbance of textural features (lamination), and frequently without pyroclastics.

The isotopic compositions of sulfur and carbon in palygorskite-bearing rocks of Holes 366,368 , and 370 provide an important additional argument. Table 8 shows that the isotopic composition of sulfide sulfur is mostly negative, about $-20 \%$. Such values are peculiar to sedimentary-diagenetic sulfides that result from sulfate reduction. They may mean that sulfides appeared in the uppermost layer of sediments under conditions of free exchange of the oozy and bottom water; i.e., sediments may have inherited sulfide sulfur from the moment of their accumulation, at the earliest stages of diagenesis.

Despite low concentrations of carbonates in palygorskite-bearing clays, we attempted to determine the isotopic composition of the carbonate (Table 9). Some carbonate has carbon with an isotopic composition that is lighter than normal sea carbonate. The latter is characterized by the value $\delta^{13} \mathrm{C}=0$, whereas $\delta^{13} \mathrm{C}$ of the organic matter is approximately $-25^{\circ} \%$ (Table 9). Table 9 shows the data on Hole 368 and, for comparison, those on core material from Hole 366, which contains considerable amounts of carbonate. When treated with hydrochloric acid, the samples from Hole 368 liberate $\mathrm{CO}_{2}$ only after the reaction vessels are heated, which suggests a dolomitic carbonate composition. The isotopic composition of carbonate carbon in Hole 368 is intermediate between extreme values of $\delta^{13} \mathrm{C}$ representative of normally sedimentary rocks and biogenic carbonates. This means that the carbonate carbon includes considerable carbon from organic matter.

Pyritization and carbonation (dolomitization) of the sediments are related to diagenetic, and evidently to early diagenetic, transformations. We found no indications of hydrothermal activity in the isotopic compositions of sulfur and carbon.

Such observations on genesis of palygorskite and sepiolite do not deny, however, local processes of transformation of magnesian silicates closely related to volcanism and activity of hydrothermal solutions. For example, the transformation of volcanogenic montmorillonite into palygorskite may occur in interstitial waters with a high content of magnesium cations.

Another type of palygorskite formation is related to the occurrence of palygorskitic veins in sediments and as coating on manganese nodules. And finally, Bonatti and Ioensuu (1968) have described a third type: formation of palygorskite under the influence of hydrothermal solutions during serpentinization of iron-rich minerals in Atlantic abyssal sediments.

With all this in mind, however, we cannot deny the role of chemogenic sedimentation and chemogenicdiagenetic formation of magnesian silicates and fluvial or eolian transport of these minerals from arid zones into oceanic sediments.

\section{OTHER CLAY MINERALS}

Montmorillonite occurs in rock facies reflective of quiet sedimentation by weak currents (Millot, 1964) (Hole 368, Upper Cretaceous; Hole 366, lower Eocene). It results from weathering of soils in tropical areas with long dry seasons. In the tropical dry climate, soils with montmorillonite are produced in poorly drained depressions by interaction of silica, alumina, and magnesium liberated by hydrolysis during hot humid periods. Millot (1964) points out, however, that such formation of montmorillonite was, in principle, possible not only on the continent but directly in those sedimentary depressions where molassic sediments were formed.

Kaolinite in the Leg 41 sediments resulted from tropical weathering processes and subsequent fluvial and/or eolian transport. The hydromica is mostly dioctahedral illite without expanded packets and is probably detrital. The chlorite may have resulted from washout of magmatic rocks or outflow from arid zones; it is not well preserved during laterization. The mixedlayer minerals are of two types: illite-montmorillonite and chlorite-montmorillonite. The illite-montmorillonite gives asymmetric X-ray diffraction peaks: at $13.6 \AA$ (untreated); at $17.3 \AA$ (treated with glycol); and at $9.9 \AA$ (heated to $550^{\circ} \mathrm{C}$ ). The chlorite-montmorillonite shows peaks at $14.5 \AA$ (untreated); at $16.99 \AA$ (treated with glycol); and at $13.8 \AA$ (heated). Both types of mixed-layer minerals resulted from degradation of minerals on the African continent.

\section{CONCLUSIONS}

After studying mineralogy and facies types of palygorskitic and palygorskitic-sepiolitic oceanic sediments of Leg 41, we conclude the following: 
TABLE 8

Isotopic Composition of Sulfide Sulfur in the Core of Deep-Sea Holes, Hole 368

\begin{tabular}{|c|c|c|c|c|c|}
\hline $\begin{array}{c}\text { Sample } \\
\text { (Interval in } \mathrm{cm} \text { ) }\end{array}$ & $\begin{array}{c}\text { Facies } \\
\text { Type of Sediments }\end{array}$ & Associations of Clay Minerals & Age & $\begin{array}{l}\text { Concentration } \\
(\mathrm{mg} / \mathrm{g})\end{array}$ & $\begin{array}{l}834 \\
(\%)\end{array}$ \\
\hline $29-1,68-70$ & $\begin{array}{l}\text { Red deep-sea clays } \\
\text { (quiet sedimentation) }\end{array}$ & Montmorillonite, palygorskite & & 6.5 & \\
\hline $30-2,51-53$ & $\begin{array}{l}\text { Red deep-sea clays } \\
\text { (quiet sedimentation) }\end{array}$ & Admixture of sepiolite & & 7.3 & -23.3 \\
\hline $30-2,58-60$ & $\begin{array}{l}\text { Red deep-sea clays } \\
\text { (quiet sedimentation) }\end{array}$ & $\begin{array}{l}\text { Palygorskite, admixture of } \\
\text { mixed-layer mineral }\end{array}$ & & 2.3 & \\
\hline $31-2,78-80$ & $\begin{array}{l}\text { Red deep-sea clays } \\
\text { (quiet sedimentation) }\end{array}$ & $\begin{array}{l}\text { Palygorskite admixture of } \\
\text { mixed-layer mineral }\end{array}$ & & 2.3 & \\
\hline $31-2,71-73$ & $\begin{array}{l}\text { Red deep-sea clays } \\
\text { (quiet sedimentation) }\end{array}$ & Palygorskite & & 0.9 & +15.6 \\
\hline $34-2,51-53$ & $\begin{array}{l}\text { Red deep-sea clays } \\
\text { (quiet sedimentation) }\end{array}$ & Palygorskite & & 5.5 & - \\
\hline $34-2,61-62$ & $\begin{array}{l}\text { Red deep-sea clays } \\
\text { (quiet sedimentation) }\end{array}$ & Palygorskite & & 7.4 & \\
\hline $36-3,37-39$ & $\begin{array}{l}\text { Red deep-sea clays } \\
\text { (quiet sedimentation) }\end{array}$ & Palygorskite-sepiolite & ङ్ & 42.9 & -20.2 \\
\hline $36-3,68-70$ & $\begin{array}{l}\text { Red deep-sea clays } \\
\text { (quiet sedimentation) }\end{array}$ & Palygorskite-sepiolite & 요 & 15.4 & \\
\hline $37-4,80-82$ & $\begin{array}{l}\text { Red deep-sea clays } \\
\text { (quiet sedimentation) }\end{array}$ & Sepiolite, palygorskite & 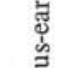 & 15.6 & \\
\hline $39-5,69-70$ & $\begin{array}{l}\text { Red deep-sea clays } \\
\text { (quiet sedimentation) }\end{array}$ & Sepiolite, palygorskite & ठ্. & 1.3 & -23.4 \\
\hline $40-3,68-70$ & $\begin{array}{l}\text { Red deep-sea clays } \\
\text { (quiet sedimentation) }\end{array}$ & Sepiolite-palygorskite & 芯 & 3.6 & 901 \\
\hline $41-2,61-63$ & $\begin{array}{l}\text { Red deep-sea clays } \\
\text { (quiet sedimentation) }\end{array}$ & Sepiolite-palygorskite & 苟 & 13.6 & -22.2 \\
\hline $41-2,70-70$ & $\begin{array}{l}\text { Red deep-sea clays } \\
\text { (quiet sedimentation) }\end{array}$ & Sepiolite-palygorskite & & 4.6 & -22.2 \\
\hline $42-2,62-63$ & $\begin{array}{l}\text { Red deep-sea clays } \\
\text { (quiet sedimentation) }\end{array}$ & Sepiolite, palygorskite & & 14.5 & -24.9 \\
\hline $44-4,68-70$ & $\begin{array}{l}\text { Red deep-sea clays } \\
\text { (quiet sedimentation) }\end{array}$ & Sepiolite, palygorskite & & 3.6 & \\
\hline $45-4,84-96$ & $\begin{array}{l}\text { Red deep-sea clays } \\
\text { (quiet sedimentation) }\end{array}$ & Sepiolite, palygorskite & & 12.5 & -28.4 \\
\hline $45-4,91-92$ & $\begin{array}{l}\text { Red deep-sea clays } \\
\text { (quiet sedimentation) }\end{array}$ & Sepiolite, palygorskite & & 14.4 & -27.9 \\
\hline $46-4,60-61$ & $\begin{array}{l}\text { Red deep-sea clays } \\
\text { (quiet sedimentation) }\end{array}$ & $\begin{array}{l}\text { Sepiolite, palygorskite, } \\
\text { montmorillonite, quartz }\end{array}$ & & 0.5 & -19.5 \\
\hline $47-5,69-70$ & $\begin{array}{l}\text { Red deep-sea clays } \\
\text { (quiet sedimentation) }\end{array}$ & $\begin{array}{l}\text { Sepiolite, palygorskite, } \\
\text { montmorillonite }\end{array}$ & & 13.6 & \\
\hline
\end{tabular}

Note: Analyses by V. I. Vinogradov.

TABLE 9

Isotopic Composition of Carbonates in Deep-Sea Holes of Leg 41

\begin{tabular}{lrc}
\hline $\begin{array}{c}\text { Sample } \\
\text { (Interval in cm) }\end{array}$ & $\begin{array}{c}\text { Concentration } \\
\text { of } \mathrm{CO}_{2}(\mathrm{mg} / \mathrm{g})\end{array}$ & $\begin{array}{l}{ }^{13} \mathrm{C} \\
(\%)\end{array}$ \\
\hline $368-27-3,41-43$ & 3.4 & -13.8 \\
$368-30-2,51-53$ & 1.5 & -18.9 \\
$368-34-2,51-53$ & 15.3 & -12.4 \\
$368-36-2,79-81$ & 3.4 & -14.9 \\
$368-37-4,71-73$ & 1.0 & -18.0 \\
$368-45-4,84-86$ & 4.4 & - \\
$366-2-3,82-84$ & 70.4 & +4.2 \\
$366-3-3,70-72$ & 169.2 & +3.5 \\
$366-7-3,72-74$ & 190.7 & +3.3 \\
$366-11-1,50-52$ & 166.5 & +3.2 \\
$366-19-1,105-107$ & 132.0 & +4.2 \\
$366-21-2,61-63$ & 198.0 & +2.8 \\
$366-24-2,106-108$ & 52.8 & +2.7 \\
$366-40-3,74-76$ & 165.0 & +3.1 \\
\hline
\end{tabular}

1) Three genetic types of magnesian silicate rocks have been distinguished. a. The first type is pelagic palygorskitic-sepiolitic clay with dolomite, but without terrigenous or volcanogenic material. Formation occurs by chemogenic-diagenetic processes in the pelagic zone of the ocean adjacent to a continent with tropical or arid climate. The zone is characterized by an abnormally intense inflow of magnesium [apparently in the form of $\left.\mathrm{Mg}(\mathrm{OH})_{2}\right]$ and silica from the areas of laterite formation, and by irregularly high heating of oceanic waters.

b) The second type is palygorskite-bearing clay and claystone with interbeds of silty material, frequently contains mica, montmorillonite, chlorite, or kaolinite. This type formed under the influence of suspension currents transporting deltaic sediments from the Senegal River and an underwater delta or canyon in the vicinity of Hole 370 .

c) The third type is pelagic limestone and nannofossil marl with interbeds of palygorskitebearing clay material. The clay fraction $<1 \mu \mathrm{m}$ is polymineral, and includes palygorskite, montmorillonite, mixed-layer minerals, kaolinite, and 
quartz. Formation of this type of palygorskitebearing deposit took place in carbonate pelagic sediments near drainless parts of the arid continent; particles of palygorskite were supplied by eolian transport.

d) On the basis of Leg 14 cores, a fourth genetic type of palygorskitic-zeolitic clays, not observed by us in Leg 41 sediments, may result from diagenetic transformation of volcanic ash (von Rad and Rösch, 1972).

2) On the basis of new data, fluvial and eolian transport of palygorskite-sepiolite particles seems possible, as does redeposition of the particles. Our conclusions are in good agreement with Wirth's (1968) data on eolian transport of palygorskite from the arid Senegal River area. The source for palygorskite in the Red Sea was also shown by Müller (1961) to be continental, as was that for palygorskite in the Persian Gulf (Hartmann et al., 1971). The occurrence of palygorskite and sepiolite in Upper Cretaceous and Paleogene sediments only, and their paragenesis with authigenic clinoptilolite and with slowly deposited clays about 20-25 m.y. old confirms the diagenetic origin of these magnesian silicates (von Rad and Rösch, 1972). Palygorskite and sepiolite probably are not forming at present. The occurrence of palygorskite in the surface layer of Atlantic Ocean sediments would seem to suggest their formation in oceanic sediments near the continents, but it is more likely a result of fluvial or eolian transport.

3) Palygorskite is detrital, and its quantitative increase toward the continent cannot always be observed, depending on the submarine topography and the nature of suspension currents.

4) We agree with von Rad and Rösch (1972) that a surplus of cations in interstitial waters is necessary for optimal chemogenic or chemogenic-diagenetic formation of sepiolite and palygorskite. Origins can vary extensively (Hathaway and Sachs, 1965). We recognize the inflow of silica by rivers draining the regions of laterization as a contributing factor for formation of magnesian minerals in sediments of Leg 41 .

5) Most other minerals of the clay fraction of Leg 41 oceanic sediments are detrital. Kaolinite is known to abound in the crusts of laterization. Dioctahedral mica and trioctahedral chlorite are unstable in the profile of laterization, but they can remain preserved to a certain extent; this might explain their limited quantitative distribution in sediments of Leg 41 . They can become more abundant during increasing aridity. In addition, chlorite can be related to washout of old magmatic rocks of northwest Africa. Montmorillonite and mixedlayer minerals could have been brought from the lateritic areas of arid zones, or could have resulted from diagenetic alteration of volcanogenic material.

\section{REFERENCES}

Bonatti, E. and Ioensuu, O., 1968. Palygorskite from Atlantic deep-sea sediments: Am. Mineralogist., v. 53, p. 975.

Chambers, G.P., 1959. Some industrial applications of the clay minerals sepiolite: Silicates Industr., v. 24, no. 4.

Elouard, P., 1959. Etude géologique et hydrogéologique des formations sédimentaires du Guebla Mauritanien et de la Valée du Sénégal: Thèse Sci.

Goldberg, E.D. and Griffin, J.J., 1970. The sediments of the northern Indian Ocean: Deep-Sea Res., v. 17, p. 513-537.

Hartmann, M., Lange, H., Seibold, E., and Walger, E., 1971. Oberflachensedimente im persischen Golf und Golf von Oman Geologisch-hydrologischer Rahmen und erste, sedimentologische Ergebnisse: "Meteor"' Forchungsergebnisse, v. 4 , p. 1.

Hathaway, J.C. and Sachs, P.L., 1965. Sepiolite and clinoptilolite from the Mid-Atlantic Ridge: Am. Mineralogist., v. 50 , p. 852 .

Martin-Vivaldi, J. and Cano-Ruiz, J., 1956. Contribution to the study of sepiolite. pt. III: Nat. Res. Council Publ., 456.

Millot, G., 1964. Géologie des argiles: Paris (Masson et Cie).

Müller, G., 1961. Palygorskit und sepiolith in tertiären und quartären sedimenten von Hadramaut (S-Arabien): Neues Jahrb. Mineralogie Abhandl., v. 97, p. 275.

Mumpton, T.A. and Roy, R., 1958. New data sepiolite and attapulgite: Fifth Natl. Conf. Clays and Clay Minerals, Proc., Washington.

Nagy, B. and Bradley, W., 1955. The structural scheme of sepiolite: Am. Mineralogist., v. 40, p. 885-892.

Pow-foong Fan and Rex, R.W., 1972. X-ray mineralogy studies. In Hayes, D. E., Pimm, A.C., et al., Initial Reports of the Deep Sea Drilling Project, Volume 14: Washington (U.S. Government Printing Office), p. 677-726.

Preisinger, A., 1959. X-ray study of the structure of sepiolite. Sixth Natl. Conf. Clays and Clay Minerals, Proc., London.

Seibold, E. and Hinz, K., 1974. Continental slope construction and destruction West Africa: In Burk, C.A. and Drake, C.L. (Eds.), The geology of continental margins: New York (Springer-Verlag), p. 179-196.

von Rad, W. and Rösch, H., 1972. Mineralogy and origin of clay minerals, silica and authigenic silicates in Leg 14 sediments. In Hayes, D.E., Pimm, A.C., et al., Initial Reports of the Deep Sea Drilling Project, Volume 14: Washington (U.S. Government Printing Office), p. 727752.

Wirth, L., 1968. Attapulgites du Sénégal Occidental: Laboratoire Géol. Univ. Dakar, Rapport no. 26. 